\title{
CIBERNÉTICA APLICADA A LA ENVOLVENTE ARTIFICIAL \\ (Trabajos con el Caos o Sistemas Complejos: \\ Vivienda Progresiva)
}

Bernardo Uribe Mendoza*

Las fotografías recientes fueron facilitadas por la Fundación Estructurar y su Directora, Ana Leonor Rueda.
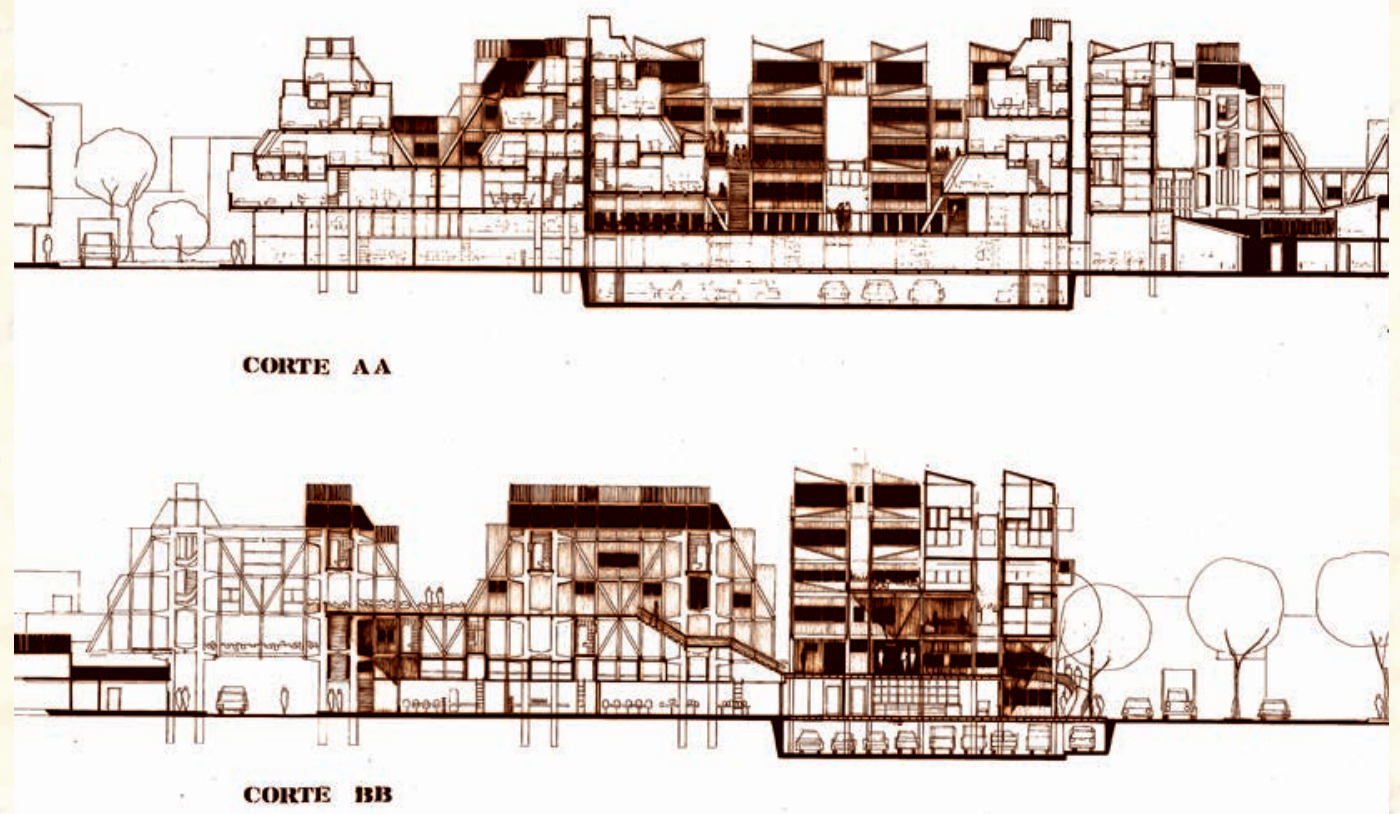

\section{RESUMEN}

El artículo desarrollado esboza la posibilidad de aplicar los estudios de sistemas de complejidad, basados en teorías contemporáneas de sistemas como las de Niklas Luhmann y otros autores, también al diseño tanto topológico como tectónico de una envolvente artificial arquitectónica. Para lo anterior se analizan experiencias académicas y profesionales sobre un tema arquitectónico y urbano en el cual estas herramientas conceptuales y pragmáticas son necesarias: la vivienda progresiva de las ciudades latinoamericanas de la $2^{\mathrm{a}}$. Mitad del Siglo XX y su recepción en los medios académicos y profesionales de la época.

\section{PALABRAS CLAVE}

Envolvente artificial, vivienda progresiva. 


\section{APPLIED CYBERNETICS ARTIFICIAL CASING (Works with Chaos and Complex Systems: Progressive House)}

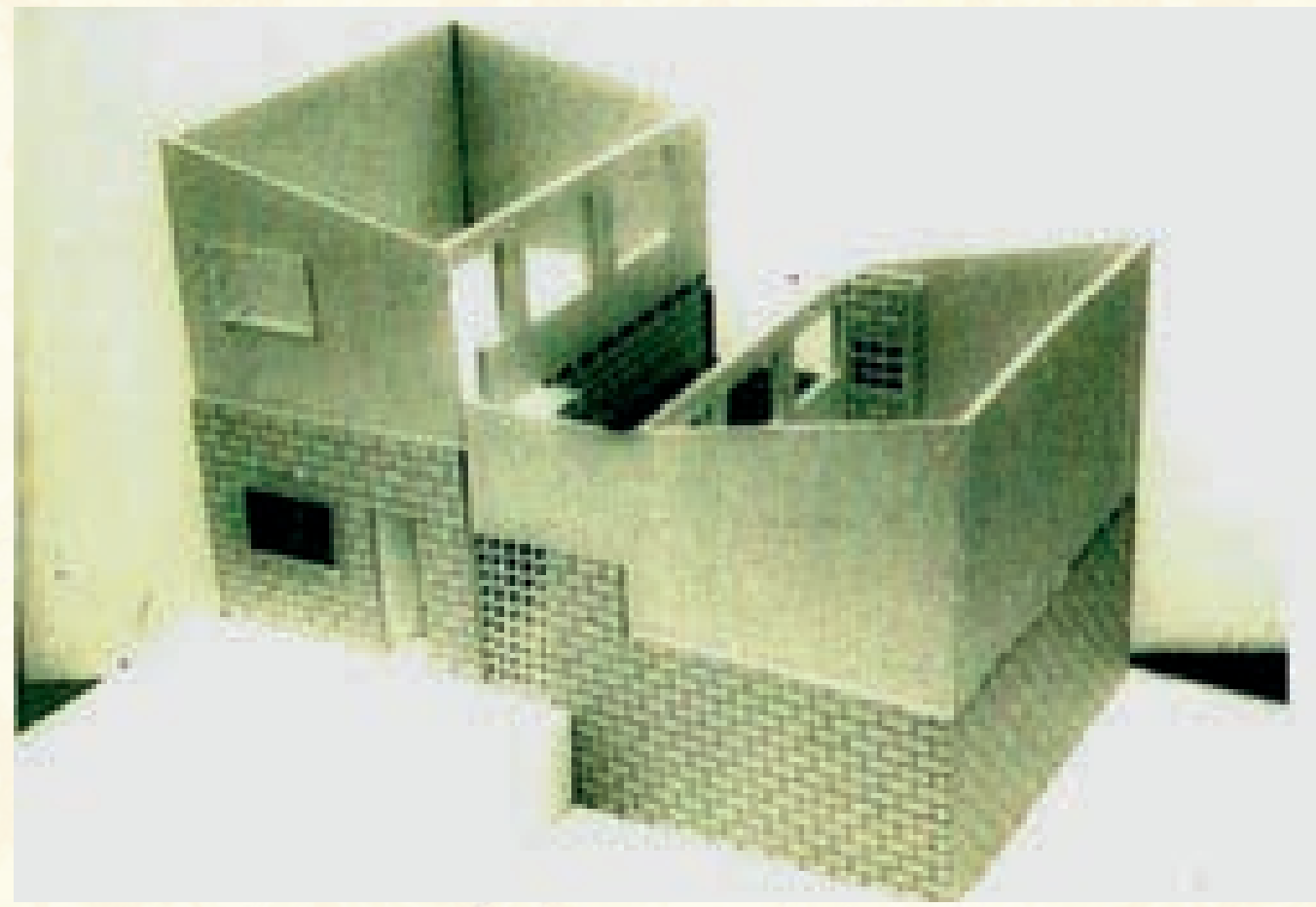

\section{ABSTRACT}

The article outlines the applicability of the studies of complex systems to design both a topological and tectonic architecture artificial surround or 'border', based on contemporary theories of systems authors such as Niklas Luhmann and others. It also discusses academic and professional experiences on a subject architectural and urban context in which these conceptual and pragmatic tools are needed: progressive housing of Latin American cities of the 2 nd. Half of the Twentieth Century and its reception in the academic and professional at the time.

\section{KEY WORDS}

Artificial surround, Progressive house. 


\section{Ejercicio teórico}

En términos amplios la arquitectura convencional es una disciplina o pragmática enfocada hacia la generación de una envolvente artificial material la cual incluye en su interior/ exterior los diversos y complejos niveles de la interacción antropológica individual y social, topográfica y geográfica, entre otras. La pragmática constructiva material de una envolvente artificial en su sentido convencional es entendida como una edilicia, en lo cual se ha hecho énfasis durante una historia de siglos / milenios en las más variadas culturas y regiones del planeta en dos grandes aspectos: a) la tectónica o la disposición artificial de la materialidad constructiva en la envolvente por medio de la cual se concreta en una realidad definida como urbana o como ambiental según que el énfasis esté en los aspectos de agrupamiento antropológico o en los de la interacción territorial con la naturaleza o la geografía aspectos que van desde la recepción y tratamiento de la luz solar hasta la forma de deponer los residuos biológicos corporales humanos, y b) la topología o el sistema formal geométrico - espacial mediante la cual se desenvuelve o se sistematiza ésta envolvente según principios que pueden ir desde el espacio trivial ( el espacio tridimensional) hasta el espacio complejo. En estos dos principales niveles de clasificación se encuentran subsumidos todos los descriptores hasta terminar por englobar la gran complejidad de los sistemas de la vida humana natural y artificial, un sistema complejo de operación en el orbe y en la urbe desarrollado por siglos y milenios, relacionado en todos estos niveles además con las capacidades artísticas, tecnológicas y científicas acumuladas en el progreso histórico en diversas culturas y cortes o cesuras históricas. Estas por otra parte, se han diversificado ampliamente en el mundo moderno, desde principios del siglo $X X$ con el surgimiento del capitalismo occidental y la proliferación de las metaurbes: diseño arquitectónico, urbanismo, historia de esta prácticas (artes y arquitectura), técnicas constructivas, economía urbana, planificación urbana y ambiental, planificación regional, entre otras.

En desarrollo de un ejercicio teórico sobre la pragmática edilicia, pero incluyendo nuevas extrapolaciones enfocadas hacia una concepción de complejidad o caótica, fue abordado entre fines de los 70 s. y principios de los 80 s. del siglo XX el tema de una vivienda progresiva en Bogotá, de una manera más bien axiomática, pues el trabajo se desarrolló por las vías de una confrontación de la temática usual del diseño arquitectónico o edilicio como éste era entendido en la época, en la cual estaban aun recientes los experimentos de diseño de los 60 s. y el movimiento megaestructural tenía aun huellas vivas, con el de una vivienda inusual o extrema, es decir un contrapunto entre la propia disciplina académica de la pragmática edilicia de la profesión de la arquitectura, o la metodología del diseño o la tipología de la vivienda como se denominaba esto al interior del oficio arquitectónico y fenómenos habitacionales extremos del momento en meta-urbes nacientes en Suramérica. Tal como sucede en el caso también de otros saberes expertos, los fenómenos y situaciones extremas convierten de facto en insuficientes o simplemente obsoletas las pragmáticas y las metodologías acumuladas con relación a estos objetos inusuales de trabajo.

Para el caso abordado de la vivienda progresiva en Bogotá, los niveles de lo tectónico y de lo topológico en una envolvente artificial edilicia son extrapolados mas allá de los habituales significados pragmáticos que estos han acumulado históricamente en la vivienda como un hábitat biológico y cultural (formal) para individuos o grupos sociales humanos. La envolvente artificial denominada como vivienda es definida habitualmente en la pragmática edilicia arquitectónica sobre todo por esquemas culturales de interacción individual / grupal los cuales involucran series de sub-sistemas como la presencia corporal (ergonomía y escala), aspectos mecánicos ( maquinaria tecnológica de estructura e infraestructura o appliances ) y aspectos de interconexión (servicios domiciliarios), todo lo anterior como micro-procesos parte de procesos o de otros subsistemas de gran escala de naturaleza 
económica / urbana, geográfica. En la compartimentación de saberes y pragmáticas imperante en la modernidad en la profesión de la arquitectura convencional, el tema de la vivienda ha sido uno de los más establecidos por diversas corrientes o teorías del diseño arquitectónico, hasta convertirse en muchos casos en signo epocal de estos diversos movimientos o escuelas de diseño.

El caso del tema de la vivienda progresiva, término acuñado por estudiosos de sociología urbana en Colombia y América Latina en los años 60 y 70, denomina un proceso en el cual el uso habitacional simple se transforma. La unidad domiciliaria se modifica en una especie de trans-vivienda, en la cual se suman a la complejidad de procesos antropológicos extremos - familiar, individual - resultado de la migración urbana y transculturación humana acelerada en asentamientos espontáneos emergentes o explosivos en las grandes capitales latinoamericanas del momento, y a la alteración radical del comportamiento social resultante, un inusual productivismo económico y material-constructivo expresado o condensado en la evolución y consolidación del predio habitacional como unidad polivalente mixta para vivir y explotar económicamente. Abruptamente la envolvente edilicia se vuelve de inerte y protectiva en activa y evolutiva, cambiante durante períodos de tiempo largos, en los cuales se disuelven y subdividen las unidades habitacionales alterando los parámetros convencionales de la vivienda denominada unifamiliar / multifamiliar, implícitamente de esta manera requiriendo una aproximación metodológica de sistemas de complejidad para la comprensión de este proceso arquitectónico, es decir de tan sólo uno de los subsistemas implicados, más aun, si al acercarse a este tema, se hace con el propósito de catalizar e intervenir sobre sus resultados finales como celda de un tejido resultante en las ciudades en las cuales se ha desarrollado.

Por las limitaciones en la aproximación analítica, muy de la época, basadas en metodologías de disección disciplinar, es decir metodologías basadas en sistemas de análisis determinísticos, el tema de la vivienda progresiva aparecía en los medios académicos y del oficio arquitectónico de ese período como una deformación de facto de la vivienda unifamiliar / multifamiliar convencional; como un resultado inevitablemente caótico formal y social de grandes contradicciones en el organismo económico-urbano de las capitales latinoamericanas, sometidas a los fenómenos de una migración y asentamiento territorial explosivo o catastrófico y a una apabullante e insuperable pobreza material resultado de éstos procesos, finalmente como algo poco comprensible y asimilable en los ámbitos establecidos del diseño o arte arquitectónico propiamente dicho, y era por ello considerado objeto de estudio sólo para los organismos de asistencia social urbana.

No obstante, en este punto, se pasaba por alto en la época, localmente, que el tema general de una trans-vivienda y las deformaciones de los esquemas individuales y grupales del hábitat o la vivienda multifamiliar había estado en boga en los centros de estudios urbanos y facultades de arquitectura durante los años 50 y 60, es decir una década antes, en los países del primer mundo, en los proyectos de las mega-estructuras urbanas, con las cuales se proponía adaptar a una era postindustrial, la ciudad moderna de fines del Siglo $X X$. Supuestamente, una movilidad individual y una descomposición del núcleo familiar predominante en la ciudad de fin de siglo, y una polivalencia cultural en las actividades urbanas incitaba a pensar la envolvente arquitectónica como colmenas migratorias de alta densidad, de servicios y de gran potencial de variabilidad, por ejemplo en el proyecto paradigmático para un Fun Palace de Konstantin Niewenhaus de 1957.

Particularmente en la variante megaestructural del metabolismo japonés (Tange, Kurokawa, Kikutake y otros )', se asignó a la envolvente espacial artificial domiciliaria humana una nueva dimensión dinámica y compleja inspirada en la metáfora de la vida o de los ciclos metabólicos como son metamorfosis, evolución, crecimiento, ciclos propios de
Choay, Francoise, Nueva Babel, en Arquitectura Viva , Revista de Arqui- tectura \# 35 Marzo/Abril , 1994,Ma- drid


2 Jencks, Charles, La Arquitectura de Futuro, Gili, Barcelona, 1973

Lynn, Gregg, Animate Form, Princeton Architectural Press, New York , 2000 los organismos vivos; las megaestructuras arquitectónicas según Tange y otros, debían ser considerados como un organismo vivo, apto al cambio y a la inclusión de procesos evolutivos y de metamorfosis de origen antropológico expresados finalmente de manera tectónica y topológica en la envolvente artificial de la ciudad, es decir de éstas mega-estructuras. Esta visión orgánica metabolista indicaba así el estudio basado en metodologías de sistemas complejos, aplicados a los términos de una pragmática arquitectónica; fue sin embargo reducida a un cinetismo formal extrapolado de la revolución maquinista avanzada de la segunda mitad del siglo $X X$, de éste modo grúas y containers ${ }^{2}$, permitirían transformar las unidades de habitación y trabajo adaptando su crecimiento al complejo organismo urbano post-industrial, logrando una movilidad geográfica del hábitat tanto individual como grupal dentro del conjunto global de un tejido urbano extendido en mega-anillos de actividad incluso de dimensión continental. La vivienda sería aquí ya no definida como unifamiliar o multifamiliar sino una extensión corporal movible del cuerpo individual y de nuevas formas grupales de los habitantes urbanos.

El grupo italiano Superestudio reduciría este cinetismo maquinal a versiones más clarividentes de la tecnología actual y concebiría estas como una segunda piel mutable elaborada con materiales artificiales electrónicos y plegables.

Sin embargo, desde el punto de vista teorético, el enfoque en la aproximación metabólica japonesa a la envolvente artificial arquitectónica ha conservado hasta el día de hoy su vigencia como una alternativa al cul de sac de una tectónica arquitectónica postmoderna y también como un innegable preludio de las tendencias organicistas actuales que han buscado, como en el caso del folding (Gregg Lynn) ${ }^{3}$, incluir en la topología de la envolvente artificial nuevas fuerzas virtuales, visualizadas y simuladas computacionalmente, añadidas como huellas materiales (tectónicas y topológicas) tales como fuerzas del sitio, del tráfico, del programa, gradientes formales, entre otras.

Es dentro del anterior contexto académico, que la vivienda progresiva, singular al mundo latinoamericano del período, invitaba a re-elaborar la noción metabolista de una envolvente arquitectónica artificial evolutiva, compleja, mutable y desarrollar una metodología de diseño arquitectónico basado en sistemas de complejidad por los ciclos de largo desarrollo y la conjunción de diversos subsistemas caracterizados por altos grados de aleatoriedad debidos a la auto-construcción artesanal usados por los inmigrantes rurales en la construcción de la vivienda en las ciudades, la aleatoreidad de los ciclos económicos, todo lo cual le confería una dimensión inédita y específica, que la diferenciaba de entrada de la gran tecnología post-industrialmaquinista del movimiento megaestructural metabolista original de la década anterior.

\section{Ejercicio Teórico de Diseño: Barrio Restrepo Bogotá}

(Ejercicio académico desarrollado para sustentar la tesis de grado en Arquitectura bajo la dirección de los arquitectos: Hernán Viecco Sánchez, Roberto Rodríguez Silva y Rafael Gutiérrez ejecutado durante el Primer Semestre de 1980. La síntesis fue presentada en el concurso de vivienda anual de la revista Japan Architect "Schinkenchiku-cha" 1980 (mención honor, jurado: Kisho Kurokawa, discípulo de Kenzo Tange y miembro destacado del grupo del Metabolismo japonés en los 60 s.) La maqueta de estudio fue elaborada por Camilo Vargas Osorno 
Fig. I. Dibujo axonométrico correspondiente al estado de consolidación de la manzana estudiada en el Barrio Restrepo de Bogotá según planos urbanos de la época y visita de campo en 1980 (color gris) y proyección de crecimiento progresivo según las variables de vivienda compartida y en arrendamiento del estudio de Reveiz et alt de 1979 (color naranja).

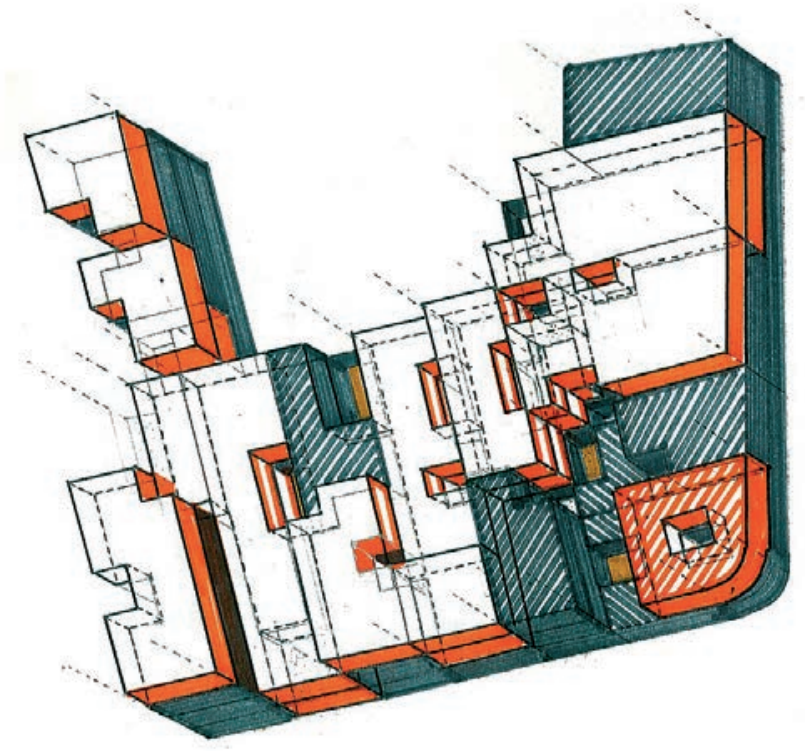

Fuente: El autor.

Según lo introducido, el tema de la vivienda progresiva, su dinámica reproductiva y de subdivisión permite ser asociado con la idea de una envolvente edilicia megaestructural del movimiento del metabolismo japonés, pero la complejidad de los fenómenos antropológicos involucrados alcanzan una dimensión de complejidad incluso mayor, por la aleatoriedad resultante de una aplicación de la autoconstrucción artesanal en el subsistema tectónico de la envolvente. La vivienda progresiva en ciudades como Bogotá, aparece como una pragmática metabólica de facto pues consiste en ampliar, construir y consolidar la envolvente arquitectónica de una unidad predial, dividiendo y subdividiendo recurrentemente las unidades de vivienda durante décadas de evolución y transformación intermitentes. La conjunción de varios sistemas y subsistemas involucrados en este proceso de transformación, tales como factores de economía urbana, factores antropológicos en diversos niveles o sub-sistemas, aspectos derivados de la tecnología y la geografía, todos además incrementados por un factor subjetivo (formal), los factores culturales introducidos mediante la autoconstrucción artesanal, solo puede ser explicado si es abordado de manera transversal y global (holística) por las teorías de sistemas complejos o del caos, las cuales en el período de trabajo del proyecto en los 70. aun no contaban con el desarrollo y las aplicaciones que tienen hoy ${ }^{4}$, tal vez sólo en las disciplinas matemáticas y la ciencia computacional había desarrollos importantes de las teoría de sistemas de autocontrol y en la biología teorías de sistemas de retroalimentación. La cibernética era entendida en la época, como una disciplina que intentaba ante todo modelar la mente humana... los trabajos de Luhmann ${ }^{5}$ sobre la aplicación de la cibernética, es decir los sistemas de control y retroalimentación, y los métodos de diferenciación y de reducción de sistemas complejos a la sociología en general y a los procesos de comunicación humana sólo aparecerían a principios de los 90 y sólo en la década siguiente se estudiaron a fondo en los medios académicos alemanes y en el momento actual encuentran difusión también en lengua inglesa.
4 Por ejemplo las teorías de sistemas y el nuevo pensamiento cibernético alemán en Günther y Luhmann sólo hasta el momento actual está encon- trando recepción en los estudios cul- turales, teoría de medios y diversas disciplinas relacionadas con la soci- ología aplicada. Este nuevo enfoque de la teorí de sistemas, pasando por (asto por sus implicaciones epistemológicas ha sido extrapolado también a prácticas artísticas.
5 Luhmann, N, Social System, Stanford University Press,Stanford, 1995.


6 Reveiz, E., et alt , Vivienda Compartida y en Arrendamiento en Ciudad Kennedy (Etapas I,2 y 3) CEDE, Centro de Estudios de Economía, Universidad de los Andes, Bogotá, 1974-9.
Por lo anterior, de manera comprensible, en los 70. el enfoque académico metodológico al tema de la vivienda progresiva se dio por separado: por un lado estaban los sociólogos o economistas urbanos como Zorro y Reveiz ${ }^{6}$ quienes definieron un modelo matemáticoeconómico por medio de matrices y de teorías matemáticas llamadas de juego mediante árboles de decisiones con lo cual intentaban representar, hoy escribiríamos similar, las variables económicas del proceso auto-reproductivo y evolutivo material espacial de la envolvente construida del hábitat. En este modelo económico se representaba la vivienda construida como un stock o capital acumulado para renta y ampliación y el proceso de consolidación como un modelo de decisión. El proceso de la vivienda progresiva en el subsistema económico era definido de este modo por medio de variables como la de consumo del espacio habitacional de la familia de propietarios, por unos agentes económicos de un grupo social definido como inquilinos o inmigrantes urbanos, y por unos servicios habitacionales, tales como dormitorio, lavadero, sanitario, ducha, lavamanos, cocina, escaleras comunes, entre otros servicios.

Simultáneamente, pero por separado en el campo arquitectónico se transcribía y extrapolaba este proceso evolutivo aplicando esta mecánica reproductiva en la envolvente artificial mediante sistemas de tectónica serial o de adición de los componentes de la vivienda; la envolvente espacial desde el enfoque tipológico arquitectónico se definía como la generación de un tipo habitacional bifamiliar / bifamiliar extendido y se definían los espacios como múltiples.

Por su parte los antropólogos evaluaban el comportamiento grupal de individuos y familias al interior del ambiente arquitectónico y material resultante en total, los resultados formales urbanos eran ásperos y contundentes pero inexplicables y menos asimilables en una idea de ciudad moderna organizada y funcional a la vez

que diferenciada formalmente, los barrios de vivienda progresiva no obedecían a principios o cánones de composición formal o de diseño urbano y por ello hasta el día de hoy son descritos como una parte informal, además muy notoria, de la ciudad contemporánea suramericana.

En el ejercicio académico sobre vivienda progresiva desarrollado como tesis de grado para una manzana del Barrio Restrepo, se propuso una extrapolación de la mecánica de la ampliación y la subdivisión de la unidad predial en la vivienda progresiva, según los modelos de ampliación de los estudios de Reveiz, a los niveles posibles con las tecnologías o tectónicas y topológicas mega-estructurales (acero, estructuras tensadas) propias del Metabolismo japonés, pero además adaptando éstos subsistemas tectónicos y topológicos a sistemas de autoconstrucción avanzada, los cuales eran de amplia difusión en los 70 sobre todo en el primer mundo. De allí surgieron ideas tales como las de proyectar piezas seriales en forma de estructuras colgantes para facilitar la adicción y subdivisión de la unidad habitacional (algunos de estos sub-sistemas tectónicos se han popularizado en la auto construcción en Bogotá después de los 90 , después de la llamada apertura económica en el país. En los años recientes se popularizó importar componentes en acero para la construcción, pero ésta era completamente inusual en Colombia en los 70 y 80 ).

El tema mega-estructural aplicado a la vivienda progresiva tal como fue abordado para el barrio Restrepo en 1980, no incluyó el estudio de las variables de dotación (appliances) los cuales habían sido de gran difusión en la corriente mega-estructural en Archigram, Superestudio o el Metabolismo japonés en las dos décadas anteriores. 
Tabla Gráfico I. Cuadro Superior esquema de subdivisión de la envolvente espacial en unidades, sub-unidades habitacionales y servicios domiciliarios para su implementación volumétrica en el ejercicio a desarrollar. Representación mediante las unidades de espacio definidas en el estudio por Reveiz y Zorrro, Cuadro inferior: Resultado acumulado de un período de evolución del predio según árbol de posibilidades de la mecánica reproductora de una envolvente espacial para vivienda según el estudio de Reveiz y de Zorro. (extrapolo como representacion to posibles del modelo económico para el lugar según el estudio desarrollado originalmente para el caso específico de Ciudad Kennedy ) Según el 'árbol de decisiones', se estudia la envolvente final consolidada mediante ampliación y renta de la unidad de vivienda.
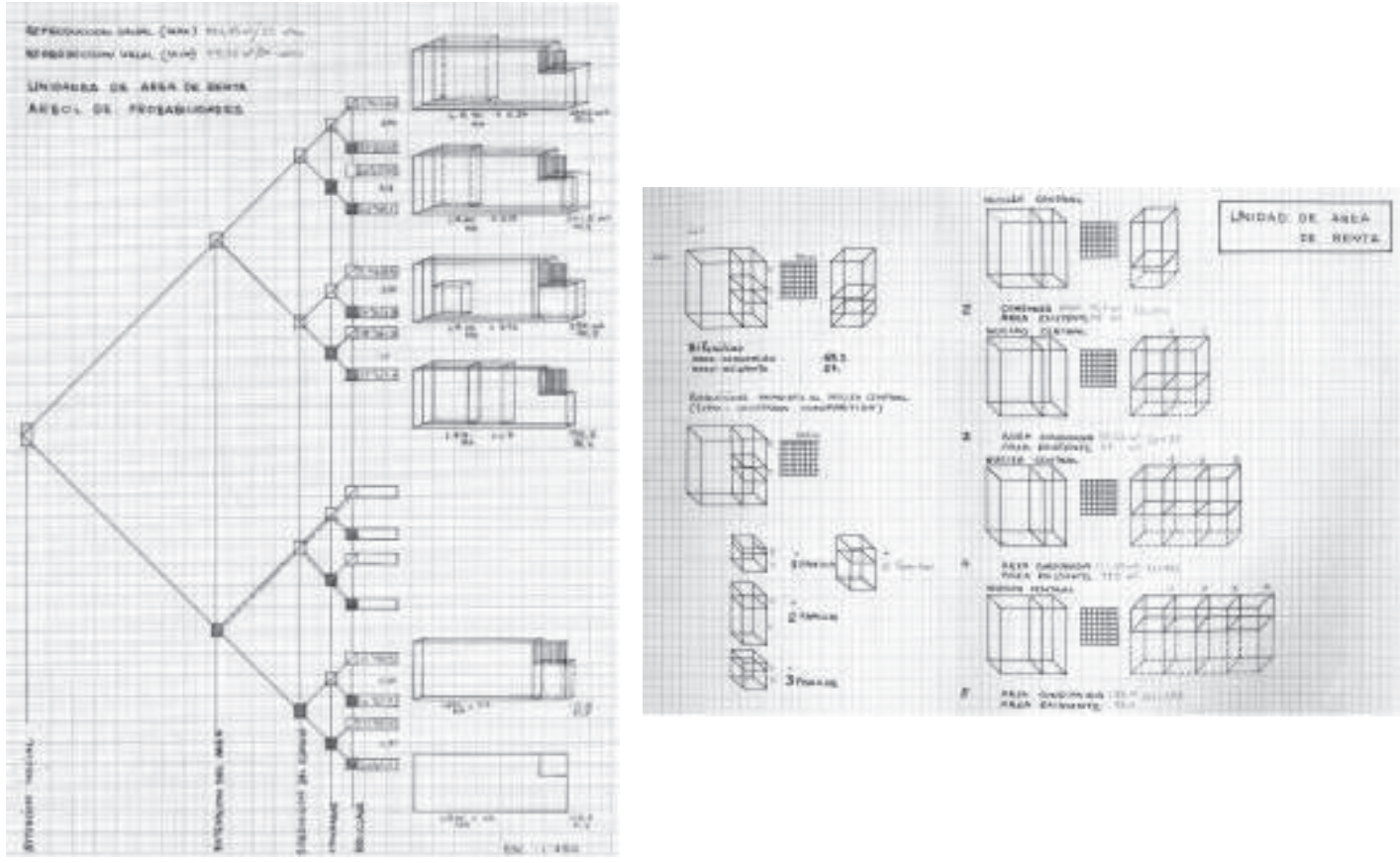

Tabla Gráfico 2. Visualización según cuadro analítico de un umbral de desarrollo de la envolvente espacial a 20 años y más allá Subdivisión potencial en varias unidades habitacionales.

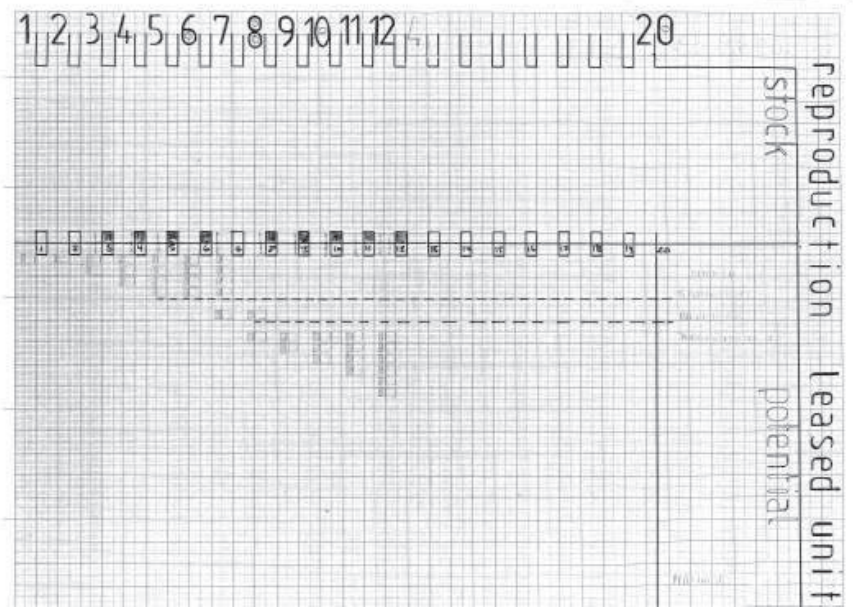

No obstante, en los dibujos artísticos - técnica de visualización del momento- se incluye de manera intuitiva el carácter aleatorio de éstos en las fases iniciales de la evolución. Muy en relación con el enfoque mega-estructural de separar la actividad urbana por niveles y de relacionar mediante co-existencia huellas o residuos tectónicos, la envolvente artificial propuesta es una resultante que evoluciona y fusiona los diversos estadios o etapas de la tectónica espacial: de la forma espontánea y aleatoria de la mecánica evolucionista artesanal de la autoconstrucción inicial a la mecánica tectónica industrial hibrida en la 
propuesta evolutiva mega-estructural (acero, partes seriadas y estandarizadas añadibles, montables y desmontables) ${ }^{7}$

7 URIBE MENDOZA, Bernardo. Racionalidad y Autogestón en Barrios Piratas: Propuesta de un Patrón de Desarrollo Progresivo, Instituto de Crédito Te ritorial, Sección Proyectos Regional Santander, Bucaramanga, 1982
Fig. 2. Dibujo correspondiente a una visión artística del desarrollo por unidad o 'predio' en la manzana estudiada en el Barrio Restrepo de Bogotá según planos urbanos de la época y visita de campo a éstos en 1980. Desarrollo Posible hasta los 20 años y más allá del desarrollo de la mecánica reproductora de la envolvente mediante tecnologías (tectónicas) más avanzadas y flexibles en acero y concreto que las artesanales empleadas

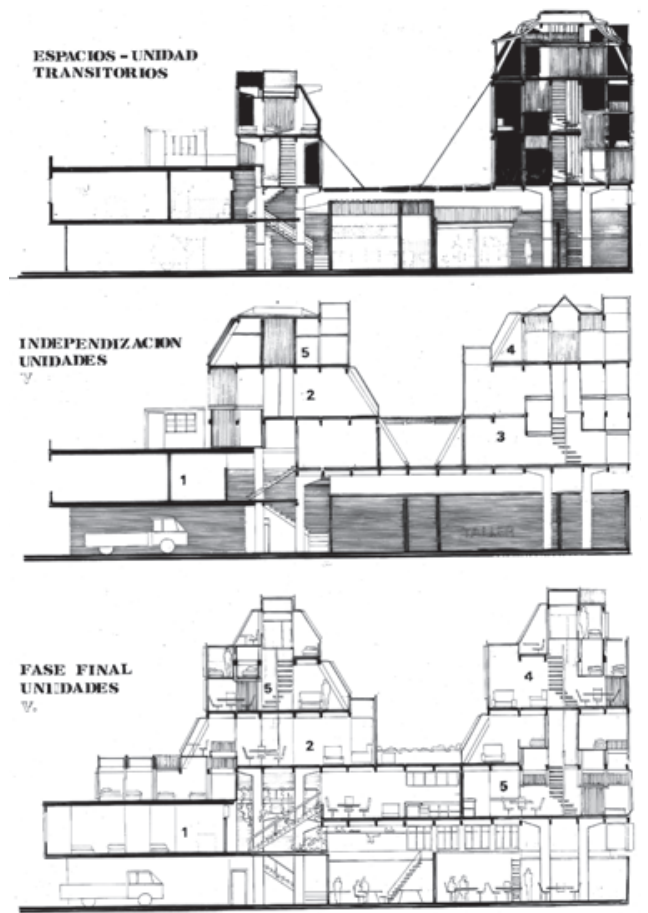

Fuente: El autor

Fig. 3. Dibujo correspondiente a una visión artística del estado de consolidación de la manzana estudiada en el Barrio Restrepo de Bogotá según planos urbanos de la época y visita de campo en 1980 a los 20 años y más allá del desarrollo de la mecánica reproductora de la envolvente.

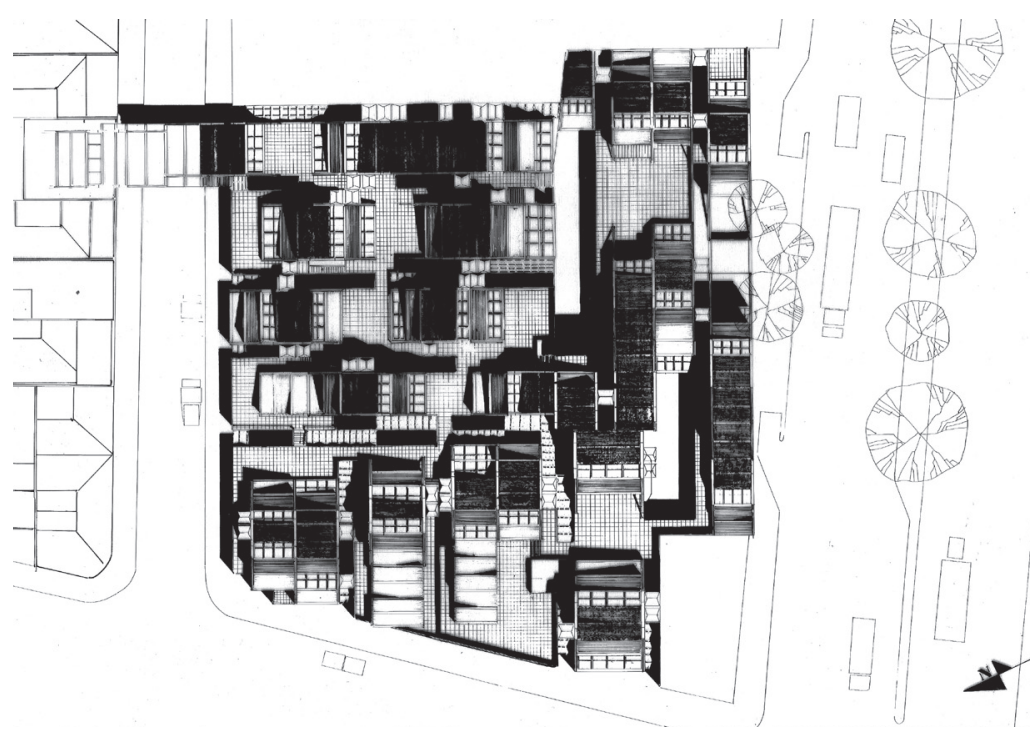

Fuente: El autor 
Fig. 4. Vistas frontales y maqueta de una visión artística del estado de consolidación de la manzana estudiada en el Barrio Restrepo de Bogotá según planos urbanos de la época y visita de campo en 1980 a los 20 años y más allá del desarrollo de la mecánica reproductora de la envolvente.

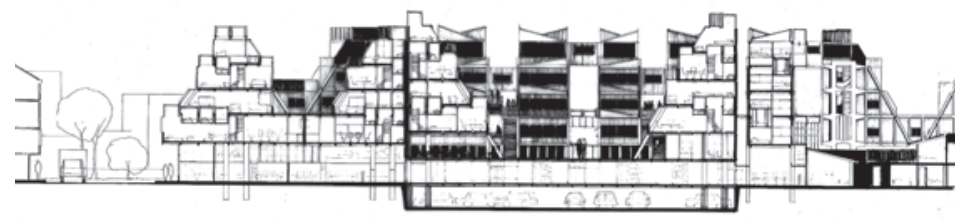

CORTE AA

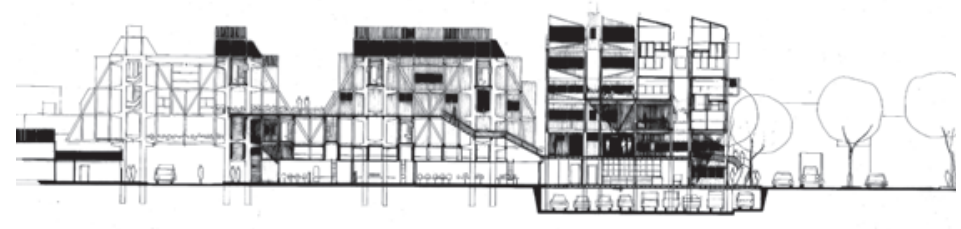

CORTE BB

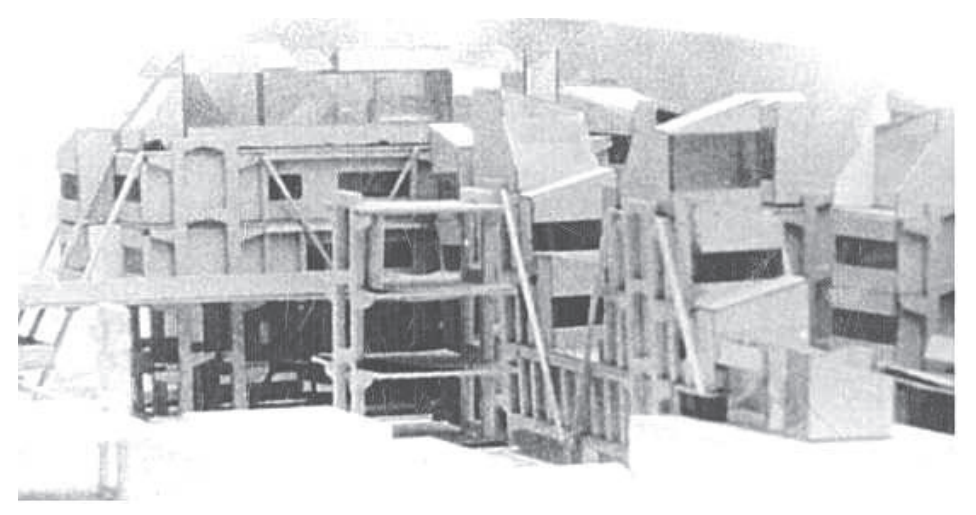

Fuente: El autor

\section{Construcción e Implementación de un Prototipo de Experimentación: Barrios Subnormales. Escarpa de la meseta de Bucaramanga. Barrio "Si se Puede"}

(El proyecto fue desarrollado y construido entre 198I-84 en la Sección de Proyectos de la Regional Santander del Instituto de Crédito Territorial, Ministerio de Desarrollo de Colombia, siendo Julio Silva Peña, director Técnico, Adolfo Enrique Nieves Valera, jefe de Proyectos y Bernardo Uribe Mendoza, arquitecto proyectista, Alonso Buitrón, Ingeniero Interventor de obra. Una proyección artística de los aspectos tectónicos de una envolvente fue elaborada para el concurso estudiantil anual de la UIA Unión Internacional de Arquitectos de 1984 con la colaboración de los estudiantes de arquitectura Luis Ardila Cancino, Maritza Villabona y Leonor Candela en 1983).

Siguiendo las conclusiones de un estudio sobre la autogestión en los barrios piratas del Área Metropolitana de Bucaramanga ${ }^{8}$, se proyectó e implementó entre 1981 y 1983 el desarrollo de un asentamiento humano en las condiciones de un proceso de construcción concebido como prototipo de experimentación para una vivienda progresiva por medios de auto-construcción artesanal y de planificación inicial institucional. En las políticas de vivienda institucionales de ese tiempo, estos proyectos eran concebidos como un proceso de urbanización de predios periféricos en las grandes ciudades del país y la construcción de una unidad domiciliaria mínima con los servicios más básicos. 
Para la propuesta de diseño se dejó de lado la concepción mega-estructural tectónica o industrial del tema del ejercicio académico pensado para el barrio Restrepo en Bogotá, pero se conservó la concepción evolutiva metabólica de los ciclos de evolución y subdivisión de la envolvente y de las unidades y grupos humanos cubiertos por ésta, en el planeamiento del sistema infra-estructural, en el plano de superficie de la implantación topográfica de las unidades prediales y del manzaneo, y en la previsión de las metamorfosis del uso domiciliario del espacio.

El sistema urbano de implantación predial retomó de la concepción mega-estructural, un sistema de superficie de loteo o urbanización de alta densidad y para evolución predial desarrollado en el concurso internacional promovido por el Gobierno de Perú en 1968 denominado Previ-Perú , concurso en el cual tomaron parte entre otros, algunos de los integrantes creadores del movimiento mega-estructural de la época, entre ellos James Stirling. El objetivo del concurso fue proponer modelos de urbanización domiciliaria de alta densidad para baja altura de construcción, aunque no necesariamente niveles artesanales de construcción.

Tabla Gráfico 3. Esquema de subdivisión predial facilitando según el árbol de posibilidades de la mecánica reproductora de la vivienda progresiva los módulos de renta y habitación al disgregar la unidad en 2 módulos de evolución separados por patio interior.
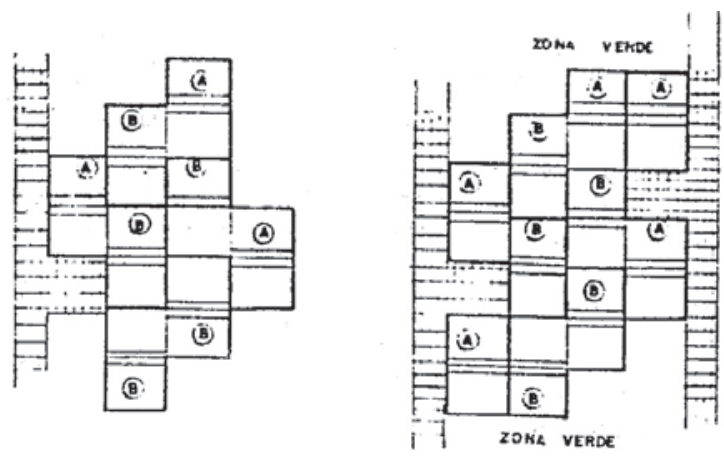

Fuente: El autor

Tabla Gráfico 4. Aspectos formales topológicos de la envolvente y la subdivisión predial facilitando el desenvolvimiento topográfico y ambiental.
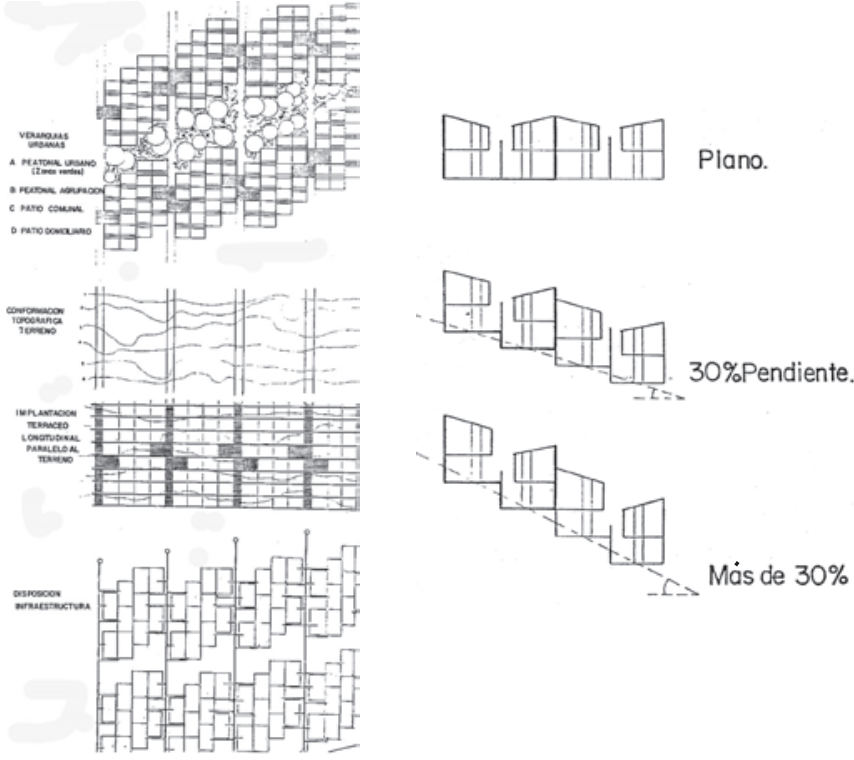

Fuente: El autor 
Fig. 5. Maqueta de estudio representa la dotación predial mínima a ser construida por el ICT y la evolución de la envolvente edilicia en los 20 años previstos para la consolidación de la unidad predial, subdivisión en varias unidades habitacionales grupales, y saturación de los potenciales de construccion mediante la tecnología de auto-construcción y subdivision de una 'vivienda progresiva'.
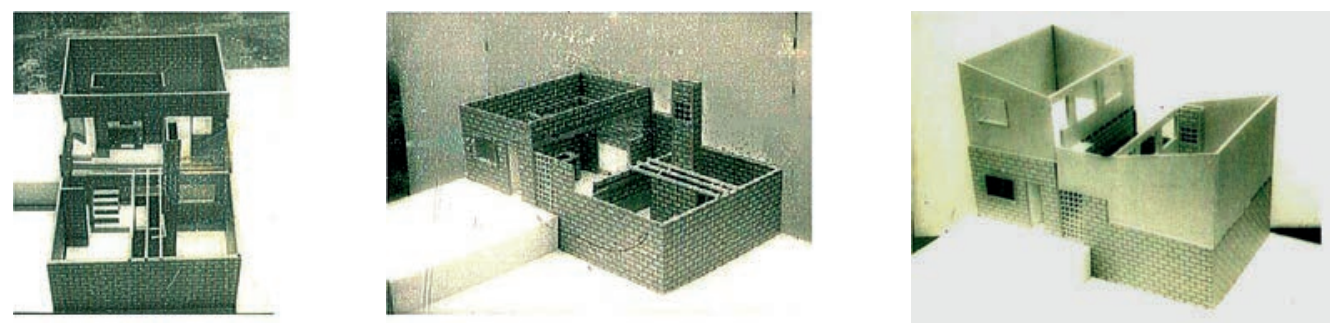

Fuente: El autor

Fig. 6. Cuadro de estudio de formas artesanales de la envolvente como fachadas y algunos de los 'appliances' como muebles de cocina y servicios.

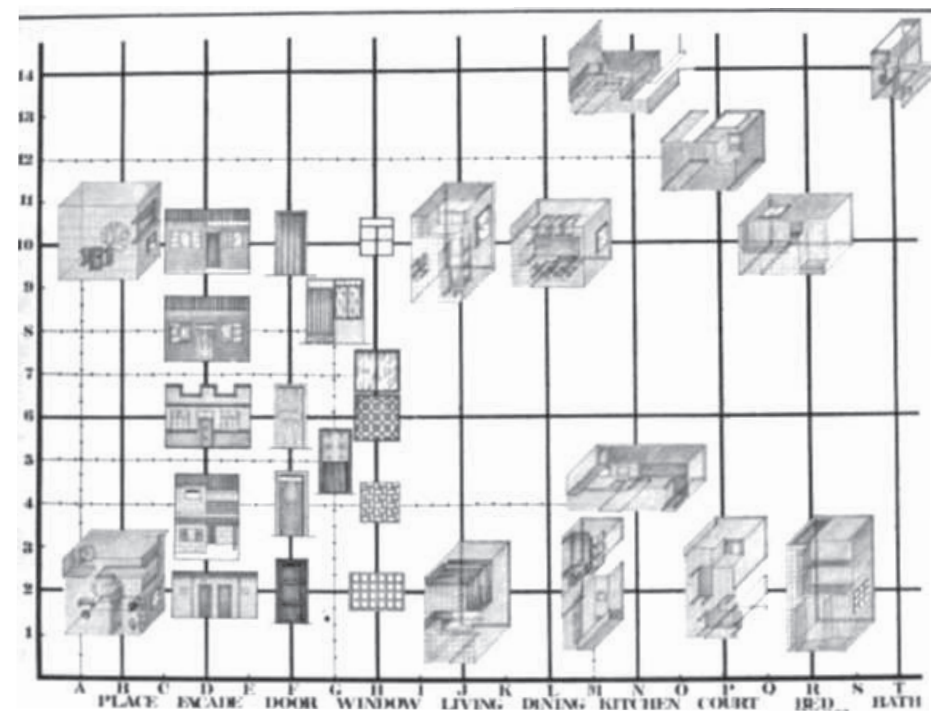

Fuente: El autor

Tabla Gráfico 5. Dibujo perspectivo isométrico mostrando aspectos formales topológicos de la envolvente y la subdivisión en el ciclo de 20 años previsto incluyendo la preservación de los patios interiores comunes y el desarrollo de terrazas.
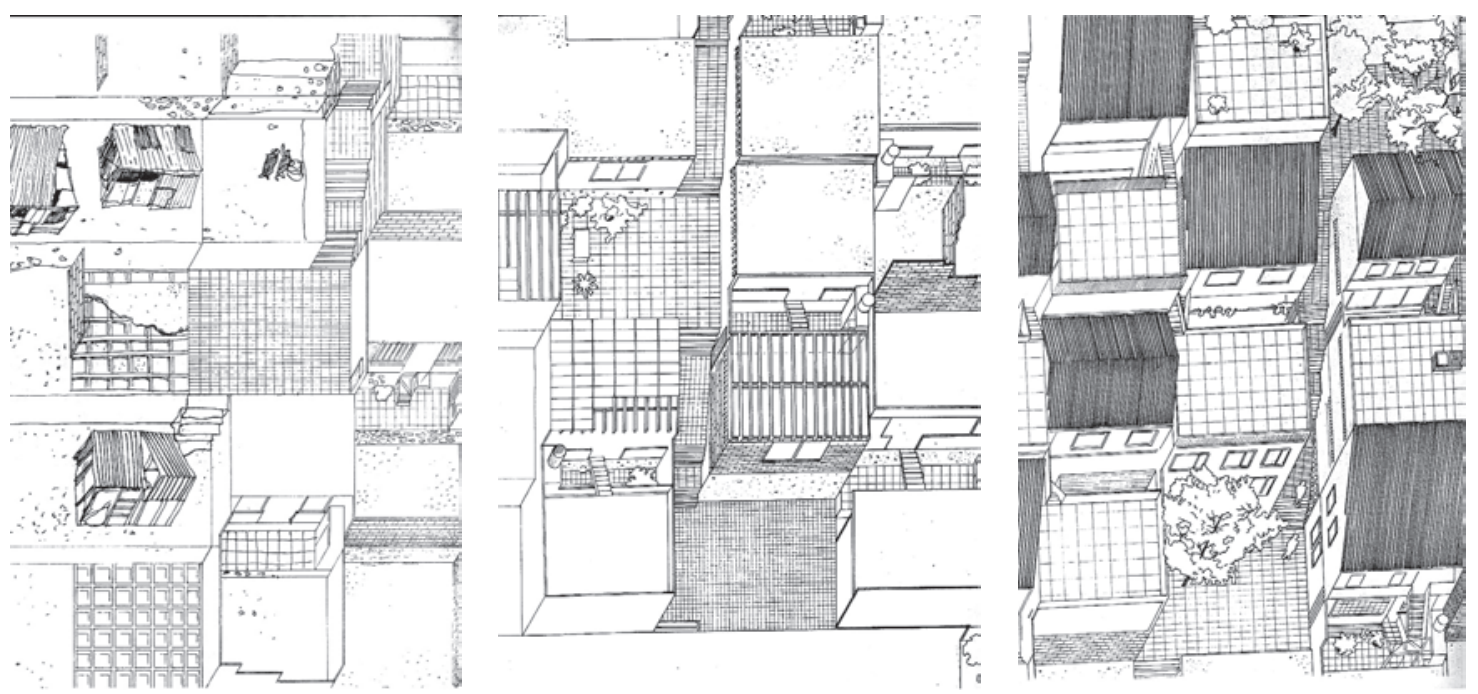

Fuente: El autor 
Fig. 6. Idea artística de los estudiantes de arquitectura sobre la evolución de los componentes tectónicos durante un período de 20 años, el cual se inicia con la construcción de unidades múltiples en los $2^{\circ} \mathrm{s}$ pisos y con la instalación artesanal de acabados tales como carpintería metálica y ventanerías durante la consolidación del barrio.

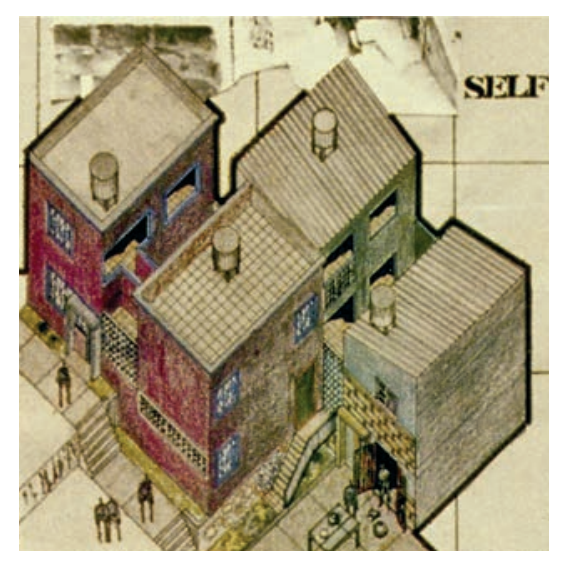

Fuente: El autor

Gráfico 6. Dibujo de localización del asentamiento en la escarpa de la meseta de Bucaramanga y Cuadros con las proyecciones del ciclo de evolución de la vivienda progresiva. Del año I a 15 años y más allá.
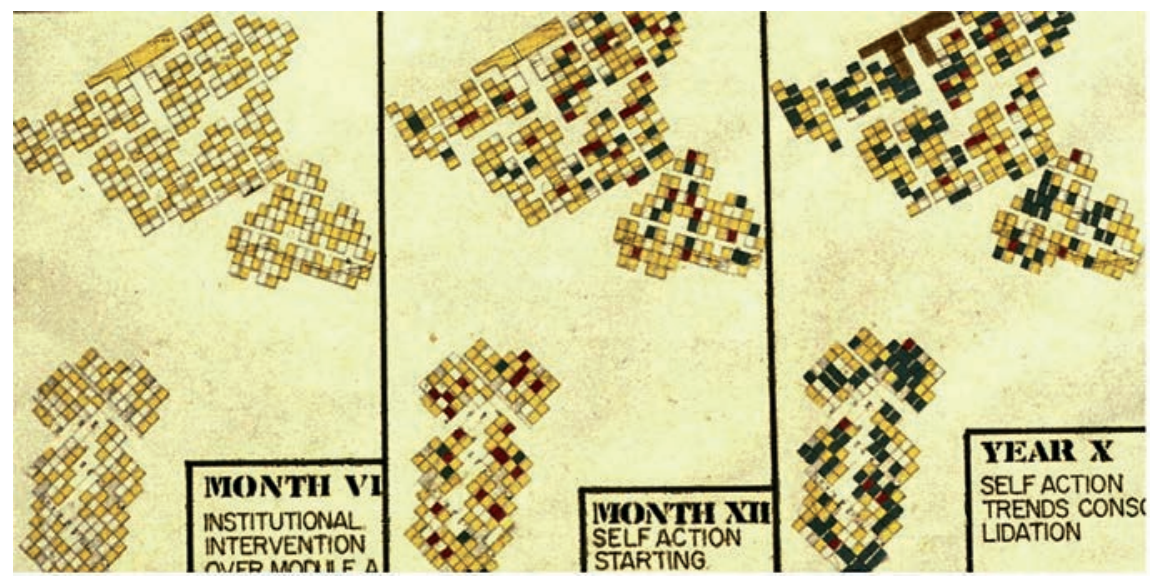

Fuente: El autor

Fig. 7. Trabajo de implantación en el lote, situado en las inmediaciones de la escarpa norte de la meseta de Bucaramanga. Terrazeo efectuado mediante maquinaria para absorber en módulos en la unidad predial, el fuerte desnivel de los terrenos, sin construir muros de contención especiales o rellenos.

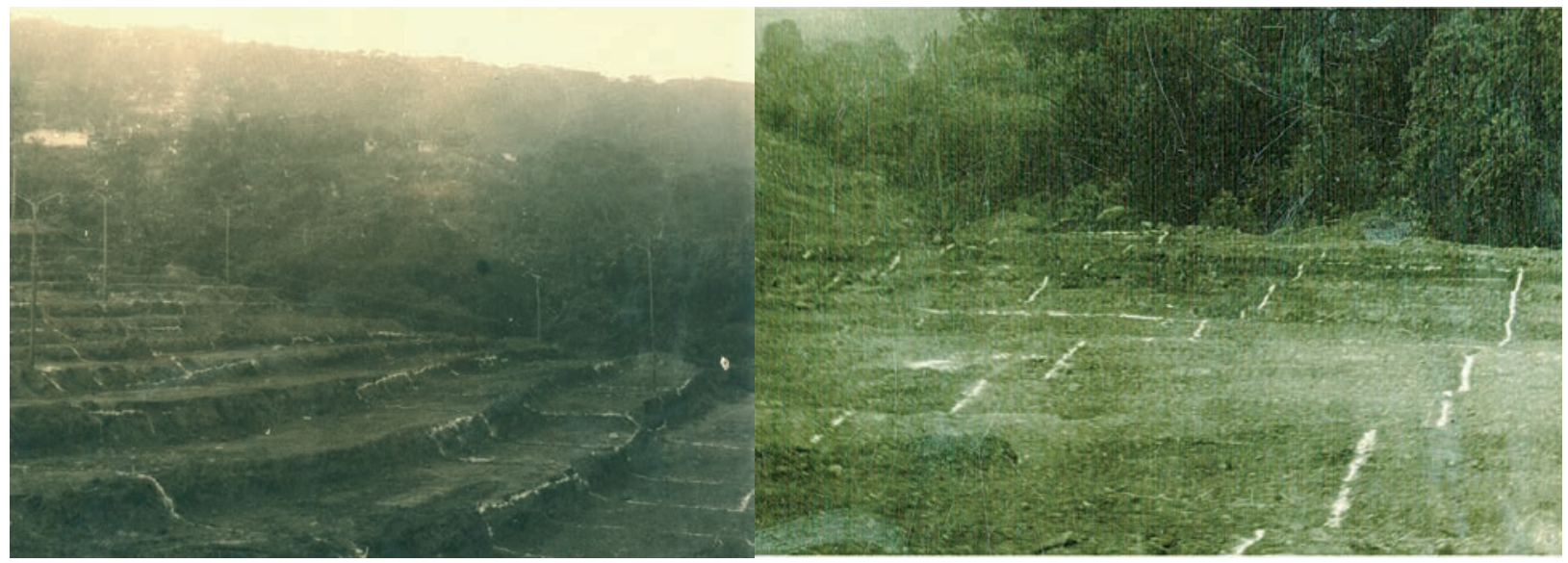

Fuente: El autor 
Fig. 8. Implantación transitoria de las familias en I de los 2 módulos de cada predio en el lote, situado en las inmediaciones de la escarpa norte de la meseta de Bucaramanga, con el fin de facilitar la construcción de una unidad mínima predial por parte del Instituto de Crédito Territorial al inicio del proceso de evolución de la vivienda.

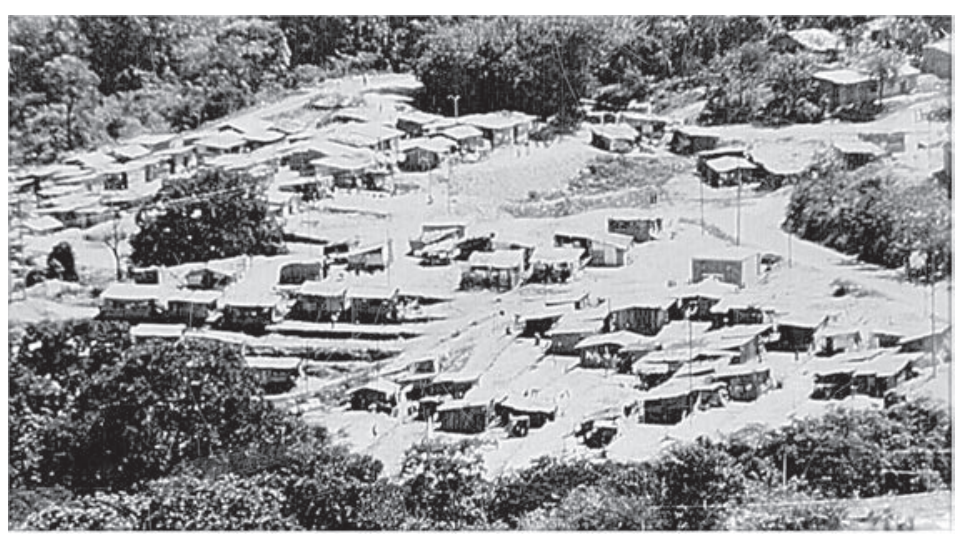

Fuente: El autor

Fig. 9. Vistas del estado del proceso de construcción en 1983 de la unidad mínima por parte del ICT Instituto de Crédito Territorial y de los 'ranchos' trasladados por los inmigrantes desde el barrio de donde fueron trasladados. La construcción de una unidad mínima predial por parte del Instituto de Crédito Territorial usando medios convencionales de baja tecnología por costos generó enormes problemas de relaciones sociales entre los equipos técnicos y los inmigrantes urbanos.

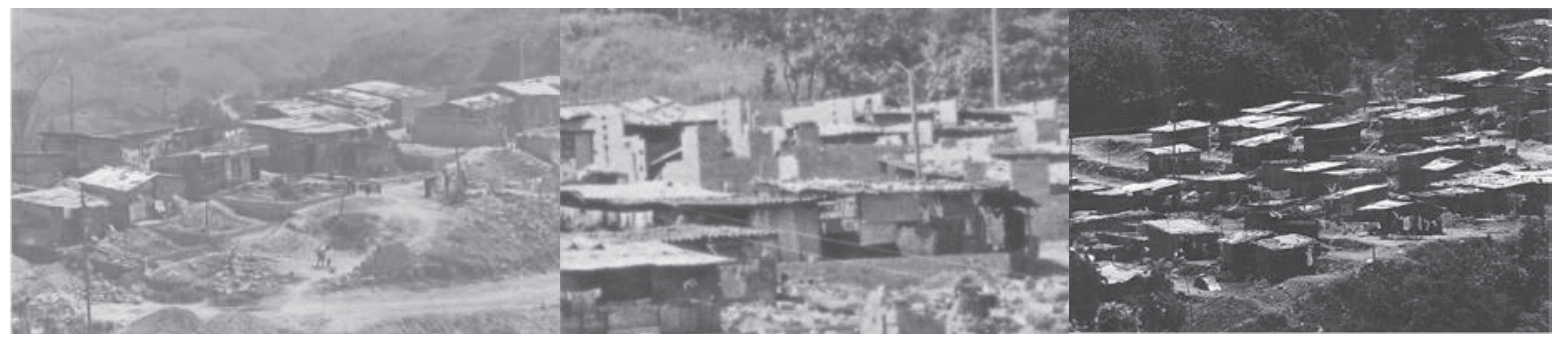

Fuente: El autor

Fig. 10. Vistas del estado del proceso de consolidación 25 años después. El proceso de construcción iniciado en 1983 de la unidad mínima por parte del ICT Instituto de Crédito Territorial, ha sido continuado por autoconstrucción por cada propietario. Comparación de la planimetría de la época y tomas de satélite en 2009 de Google. La estrategia de diseño de los patios de agrupación mostró ser efectiva pues estos se conservan como espacio público y le añaden variedad a éste, uno de los propósitos del diseño urbano de agrupación adoptado. Un trabajo de campo permitía comprobar la relación entre el grado de consolidación de la unidad predial y la renta de unidades bi y poli-familiares, según el estudio de Reveiz y Triana de 1974.

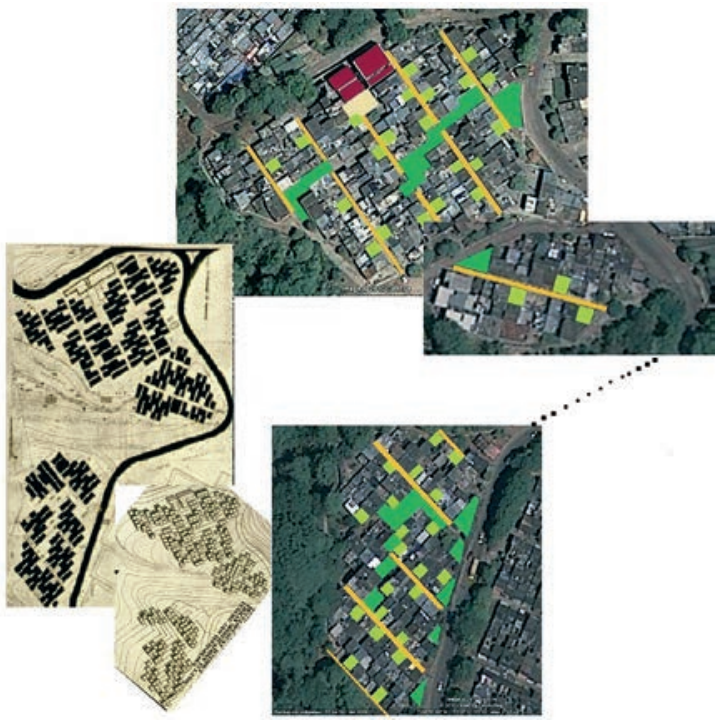


Fig. II. Vistas paisajísticas del estado del proceso de consolidación 25 años después. El proceso de construcción iniciado en 1983 de la unidad mínima por parte del ICT Instituto de Crédito Territorial, ha sido continuado por autoconstrucción por cada propietario. Esto le añade variaciones formales tectónicas al esquema topológico inicial. Las variables económicas explican el mayor o menor desarrollo de cada unidad domiciliaria. El uso inicial habitacional se mantiene y sólo algunos módulos han sido transformados en comercio zonal. No aparece el uso de los patios como interiores comunes como zona de talleres, a diferencia de lo que sucede en barrios de desarrollo urbano convencional en otras zonas de la ciudad.

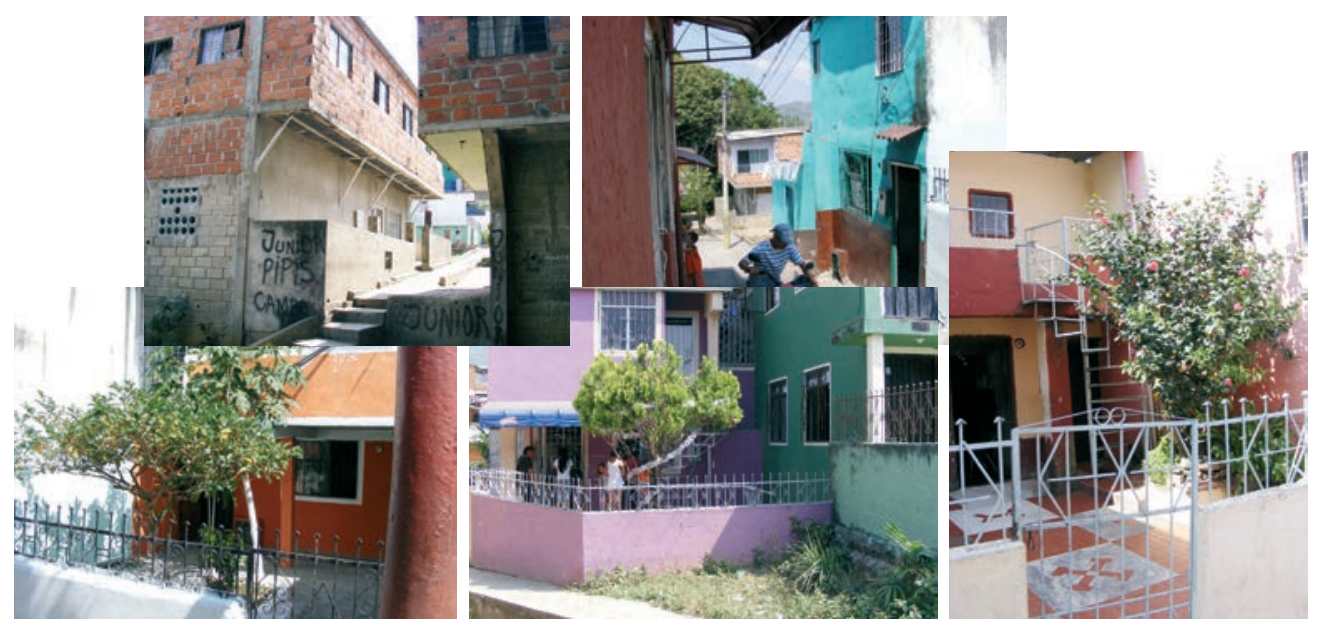

Fuente: El autor

\section{Conclusiones Preliminares}

Cuando en las grandes ciudades del país, aun en las primeras décadas del siglo XXI se continúa produciendo asentamientos semi-planeados o semi-espontáneos en predios con geografías muy semejantes a las del barrio Si se Puede de Bucaramanga, la experiencia positiva en aspectos como:

-Sistemas aleatorios de implantación topográfica para geografías escarpadas muy usuales en los centros urbanos colombianos.

- Sistemas aleatorios de diferenciación espacial tanto en los aspectos del recinto domiciliario como de la configuración o infraestructura tectónica (appliances).

- Envolvente topológica suave para interacción dinámica con cambiantes procesos antropológicos y sociológicos.

El asentamiento urbano del barrio Si se Puede ascendió a niveles cualitativos en espacio público y de agrupación, así como de consolidación física en las definiciones domiciliares a la tipificación usual de vivienda de interés social para estrato 3 según parámetros actuales en las denominaciones del mercado de vivienda urbana del país.

La experiencia desarrollada podría ser extrapolada y aumentada usando los nuevos recursos de simulación y visualización 3D digitales inexistentes en la época del proyecto. Igualmente el paradigma dominante en los estudios teóricos en el momento actual es el de la trans-disciplinareidad, el cual abre la posibilidad de integrar las diversas facetas topológicas y topográficas en proyectos como el del Si se puede.

El escenario institucional sin embargo, no es el más favorable 25 años después: el Estado desmanteló agencias como el Instituto de Crédito Territorial, o el Instituto Colombiano de Construcciones Escolares durante la Administración Barco, las cuales permitían ensayar teorías en el desarrollo y aplicación de proyectos experimentales. Lo anterior no obstante que fenómenos humanos como el abocado por los proyectos presentados en el artículo son plenamente vigentes y cubren el hábitat domiciliario de millones de personas en las grandes ciudades en el país. 
Las empresas privadas de construcción de vivienda de interés social no alcanzan a cubrir los segmentos socio-económicos inferiores de la población urbana y a diferencia del proyecto expuesto, sin una planificación comprensiva inicial, estos predios que cubren grandes áreas urbanas, han sido condenados a permanecer como focos incompletos de transvivienda sin posibilidades de consolidación o evolución.

\section{BIBLIOGRAFÍA}

Banham, Reyner, "Megaestructuras. Futuro urbano del pasado reciente “, Gustavo Gili, Barcelona, 1978

Barabasi, Axel, “Linked “, Penguin Books, London 2003

Choay, Francois, Nueva Babel, en Arquitectura Viva, Revista de Arquitectura \# 35 Marzo/Abril , 1994,Madrid

Derrida, Jacques “Las Artes espaciales” Entrevista con Peter Brunnette, "Acción

Paralela”, Barcelona, 1994

Günther, G, Das Bewusstsein der Maschinen. Eine Metaphysik der Kybernetik. Agis, Krefeld, Baden Baden 1957. 3. Auflage. 2002

Jencks, Charles, La Arquitectura del Futuro,Gili , Barcelona, 1973

Lammert et Alt ,editores Topos-Raum: Die Aktualität des Raumes in der Künste der Gegenwart, , Akademie der Kunste, 2006

Luhmann,N, Social System, Stanford University Press,Stanford, 1995

Lynn, Gregg, Animate Form, Princeton Architectural Press, New York , 2000

Mitchell, William; “City of Bits”, MIT Press, Cambridge-London 1995

Moravec, Hans, "Robot, From mere Machine to transcendent Mind", Oxford University Press

Nesbit, K, Theorizing in Architecture, The Princeton Architectural Press, New York, 1995

Noever, P, editor, Architektur am Ende ?, MAK, Prestel Verlag München, 1992 Rajchman, John, “ Constructions”, "Lightness” Pag 43-44.MIT Press, CambridgeLondon, 1998

Reveiz, E et alt , Vivienda Compartida y en Arrendamiento en Ciudad Kennedy (Etapas I,2 y 3) CEDE , Centro de Estudios de Economía, Universidad de los Andes, Bogotá, 1974-9

Wilson, Stephen, “Information Arts”, MIT, Press, Cambridge-London, 2002

Uribe Mendoza , Bernardo, Racionalidad y Autogestón en Barrios Piratas : "Propuesta de un Patrón de Desarrollo Progresivo", Instituto de Crédito Territorial, Sección Proyectos Regional Santander, Bucaramanga, 1982 